\title{
Transmission characteristics of the SO and AO Lamb waves at contacting edges of plates
}

$\operatorname{AUTHOR}(S)$ :

Mori, Naoki; Biwa, Shiro

\section{CITATION:}

Mori, Naoki...[et al]. Transmission characteristics of the S0 and AO Lamb waves at contacting edges of plates. Ultrasonics 2017, 81: 93-99

\section{ISSUE DATE:}

2017-11

URL:

http://hdl.handle.net/2433/234661

\section{RIGHT:}

(c) 2017. This manuscript version is made available under the CC-BY-NC-ND 4.0 license

http://creativecommons.org/licenses/by-nc-nd/4.0/; The full-text file will be made open to the public on 01 November 2019 in accordance with publisher's 'Terms and Conditions for Self-Archiving'., この論文は出版社版でありません。引 用の際には出版社版をご磼認ご利用ください。; This is not the published version. Please cite only the published version. 
This manuscript has been published in: Ultrasonics, Vol. 81 (2017), pp. 93-99. DOI: $10.1016 /$ j.ultras.2017.06.009

\title{
Transmission characteristics of the S0 and A0 Lamb waves at contacting edges of plates
}

\author{
Naoki Mori*, Shiro Biwa** \\ Department of Aeronautics and Astronautics, Graduate School of Engineering, Kyoto University, \\ Katsura, Nishikyo-ku, Kyoto 615-8540, Japan \\ *Present address: Department of Mechanical Engineering, Faculty of Science and Engineering, Ritsumeikan \\ University, Kusatsu, Shiga 525-8577, Japan \\ **Corresponding author. Tel: +81-75-383-3796. E-mail: biwa@kuaero.kyoto-u.ac.jp
}

\begin{abstract}
In order to gain basic insight into the interaction between ultrasonic guided waves and structural discontinuities with contacting surfaces, the transmission characteristics of Lamb waves at contacting edges of two plates are studied experimentally. The edges of two 2.5 - $\mathrm{mm}$ thick aluminum alloy plates are mated together to constitute a contacting interface of plates and subjected to different levels of compressive loading. The transmission measurements of the lowest-order symmetric ( $\mathrm{S} 0$ ) and antisymmetric (A0) Lamb modes across the contacting interface are performed in a frequency range below the cut-off frequencies of the higher-order modes. As a result, it is found that the transmission coefficient of the S0 mode increases monotonically with the applied contact pressure, but the transmission coefficient of the A0 mode exhibits non-monotonic dependence on the contact pressure and the frequency showing a local minimum. For the incidence of the S0 mode, the resonance at the contacting interface is observed as a long-time oscillation tail in the transmission waveform. The resonance frequency is found to increase with the contact pressure. The experimental results are discussed in the light of the theoretical results based on the spring-type interface model. The normal and tangential stiffnesses of the contacting interface are identified from the transmission coefficients as well as from the resonance frequency. The estimated interfacial stiffnesses increase monotonically with the contact pressure, and indicate their dependence on the frequency. Implications of the present results to the Lamb-wave based characterization of the plate contact condition are discussed briefly.

Key Words: Lamb wave; contact interface; transmission; edge resonance; interfacial stiffness
\end{abstract}




\section{Introduction}

Elastic guided waves offer an efficient means of nondestructive inspection and structural health monitoring due to their capability to propagate longer distances than bulk waves. Lamb waves, guided waves propagating in plates, have the potential to provide an effective tool for characterization of various defects in plate structures [1-5]. There have been particular interests in monitoring fatigue damage in plate structures using Lamb waves [6-8]. For example, Ihn and Chang [6] proposed a monitoring method for fatigue crack growth using a damage index based on the scattered energy of Lamb waves calculated by the short-time Fourier transform (STFT). Cho and Lissenden [8] monitored the crack growth from rivet holes based on the Lamb wave transmission characteristics. Fatigue cracks are, however, often closed with their surfaces in partial contact. In this regard, it is of primary importance to understand the interaction between Lamb waves and structural discontinuities with contacting surfaces such as closed cracks.

For the theoretical modeling, imperfect interfaces such as contacting surfaces of solids can be commonly modeled as a spring-type interface characterized by equivalent normal and tangential stiffnesses [9-14]. For Rayleigh waves, the effect of crack closure on the wave scattering response has been studied by Pecorari [15] based on this model. Recently, the interaction of Lamb waves with an imperfect joint of plates has been analyzed theoretically [16, 17]. Mori et al. [16] showed the effect of the interfacial stiffnesses on the frequency dependence of the reflection and transmission coefficients of the lowest-order symmetric (S0) and antisymmetric (A0) Lamb modes at the joint. Furthermore, Mori and Biwa [17] showed that the imperfect joint of plates exhibits resonance for the S0 mode incidence at two frequencies which depend on the interfacial stiffnesses. Based on these theoretical findings, the transmission of Lamb waves and the resonance at butt-type adhesive joints of plates were investigated experimentally [18]. It is also of high interest to examine the corresponding behavior at a butt-type contacting interface of plates as it can represent the local interaction of Lamb waves with a closed crack. In spite of this particular interest, experimental studies of the transmission characteristics of Lamb waves at such contacting interfaces have not been reported so far, while the interaction of Lamb waves with other configurations of contacting interfaces, as found in sandwiched or layered plates, has been studied by some investigators [19-21].

In the present study, the interaction of Lamb waves with a butt-type contacting interface of plates is examined experimentally. To this purpose, the edges of two aluminum alloy plates are mated together to constitute the contacting interface. The transmission coefficients of the S0 and A0 Lamb modes are measured in a low frequency range when compressive loads of different levels are applied to change the contact condition of the interface. Furthermore, for the incidence of the S0 mode, the transmission waveform is examined to investigate the resonance at the contacting interface. The experimental results are discussed in the light of the theoretical findings $[16,17]$ based on the spring-type interface model.

\section{Experiment}

\subsection{Specimens}

The schematic of the experimental setup is shown in Fig. 1. Two square A5052 aluminum alloy plates (side length $150 \mathrm{~mm}$ and thickness $2.5 \mathrm{~mm}$ ) were mated together at one of their edges to constitute a contacting interface. Two samples, denoted Sample A and Sample B, were prepared with the same size but different plate-edge surface conditions. For Sample A, the edge surfaces of both plates were polished by a \#1000 abrasive paper before being mated together. Sample B was made by polishing the edge surfaces first by a $\# 1000$ abrasive paper, then by a \#320 paper before mating them. In the ultrasonic measurements, each sample was held in stainless guide rails in order to keep the two plates in the same plane when compressive loading was applied to vary the contact condition. In addition, a single aluminum alloy plate (A5052, size $150 \mathrm{~mm} \times 300 \mathrm{~mm}$ $\times 2.5 \mathrm{~mm}$ ) was used as a reference specimen. This specimen was also kept in the guide rails to set the same configuration as the contact specimens. 


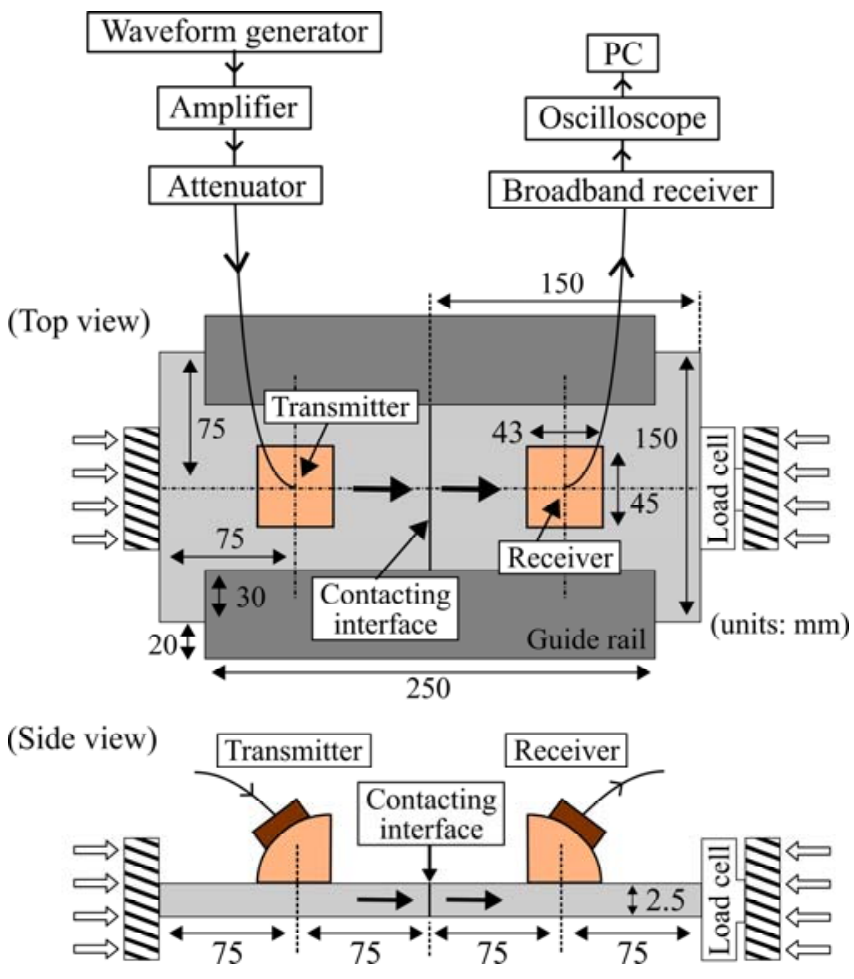

Fig. 1 Schematic of the experimental setup.

\subsection{Measurement of the transmission coefficients}

The method for the measurement of the transmission coefficients of the S0 and A0 Lamb modes has been described in Ref. [18] where it was applied to adhesive butt joints of aluminum alloy plates of the same size as those used here. As schematically shown in Fig. 1, two piezoelectric longitudinal wave transducers with nominal frequency $0.5 \mathrm{MHz}$ were fitted to adjustable-angle wedges and mounted on the surface of the specimen with liquid couplant. The distance between the two wedges was fixed to $150 \mathrm{~mm}$. A Gaussian-modulated tone-burst signal with the center frequency of $0.5 \mathrm{MHz}$ was used to excite the emitting transducer. The wedge angle $\theta$ was chosen in order to excite and detect a particular Lamb mode dominantly, i.e., $\theta=30$ deg and $\theta=60$ deg for the incidence of the S0 and A0 modes, respectively [18].

Compressive loading was applied from both ends of the sample by a clamping device, and their magnitude was measured with a load cell installed at one end. The nominal contact pressure is calculated as the measured load divided by the area of the edge face $\left(150 \times 2.5 \mathrm{~mm}^{2}\right)$. Before the ultrasonic measurements, the plates were subjected to five loading/unloading cycles up to about $8 \mathrm{MPa}$. Then the measurements of the S0 and A0 modes were performed in the subsequent loading/unloading cycles. The transmitted wave was measured at different pressures and recorded as digital data after averaging over 64 synchronized signals. The measured waveforms were then analyzed in the frequency domain by FFT (fast Fourier transform) to obtain their amplitude spectra. The amplitude transmission coefficient was obtained at each frequency by dividing the spectral amplitude by the corresponding amplitude measured for the reference plate, in the same manner as Ref. [18]. After these measurements, the measurement of the resonance effect explained below was performed for another loading/unloading cycle, and the surface roughness parameters were measured with a Taylor-Hobson surface roughness tester (Surtronic Duo). Table 1 shows the arithmetic average roughness Ra and the average maximum height $\mathrm{Rz}$ for two samples. 
Table 1 Surface roughness parameters of the specimens.

\begin{tabular}{c|cc}
\hline \hline & Ra $[\mu \mathrm{m}]$ & Rz $[\mu \mathrm{m}]$ \\
\hline Sample A & 0.40 & 2.7 \\
Sample B & 0.96 & 4.2 \\
\hline
\end{tabular}

\subsection{Measurement of the resonance effect}

The experimental study in Ref. [18] showed that the S0 mode transmission waveform at an adhesive butt joint exhibits a long oscillation tail due to the resonance effect for the S0 mode incidence. This effect is also examined here for the contacting interface of plates. The same experimental setup as described in the previous section was used, except for the transducers and the excitation frequency. Piezoelectric longitudinal wave transducers of the nominal frequency of $1 \mathrm{MHz}$ were used as the transmitter and the receiver. The center frequency of the excitation waveform was chosen to be $0.9 \mathrm{MHz}$. The wedge angle was set as $\theta=38 \mathrm{deg}$ to generate and detect the S0 mode dominantly.

\section{Results}

\subsection{Transmission coefficients of the SO and AO modes}

For the excitation of the $0.5 \mathrm{MHz}$ S0 Lamb modes, the measured transmission waveforms are shown in Fig. 2 for Samples A and B at different nominal contact pressures in the loading process, together with the waveform measured for the reference specimen. In Fig. 2, the main wave packet is located at around $110 \mu \mathrm{s}$ corresponding to the group velocity of the $\mathrm{S} 0$ mode $(5.0 \mathrm{~km} / \mathrm{s}$ at $0.5 \mathrm{MHz}$, c.f. Ref. [18]). For Samples A and B, the maximum peak of the transmitted S0 mode is smaller than that of the reference waveform, but increases with the nominal pressure.

The waveforms from $100 \mu$ s to $120 \mu$ s were analyzed by FFT to obtain the amplitude spectra. The transmission coefficient of the S0 mode is shown in Fig. 3 as a function of the frequency for Samples A and B. At each nominal contact pressure, the transmission coefficient shows flat or slightly decreasing frequency dependence. The transmission coefficients at three different frequencies $0.45,0.5$, and $0.55 \mathrm{MHz}$ are shown in Fig. 4 as functions of the nominal contact pressure in the loading and unloading processes. These coefficients are found to be increasing functions of the nominal pressure with negligible hysteresis. In Figs. 3 and 4, the theoretical predictions based on the spring-type interface model are also shown together with the experimental results, which will be discussed later in Section 4.

It is noted that the transmission coefficients of two samples shown in Fig. 4 have qualitatively different contact-pressure dependences. For Sample A, the transmission coefficient grows with higher rates at relatively low contact pressures, but appears to show saturating behavior at higher pressures. On the other hand, Sample B shows almost a linear increase of the transmission coefficient with the pressure for the whole range of the experiment. This difference should be attributed to different edge-surface polishing treatments of two samples. Smoother edge surfaces of Sample A would naturally fit together easily and transmit the ultrasound better with low contact pressures, while rougher edge surfaces of Sample B would resist tight fitting and ultrasound transmission. It is, however, noticeable that Sample B shows higher transmission than Sample A near the maximum contact pressure of $8 \mathrm{MPa}$. The background for this reversal is not clear, but should also be due to the specific surface preparation of the two samples. 
(a)

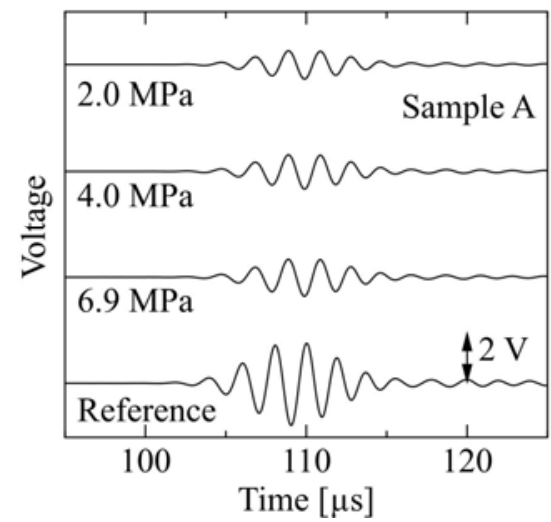

(b)

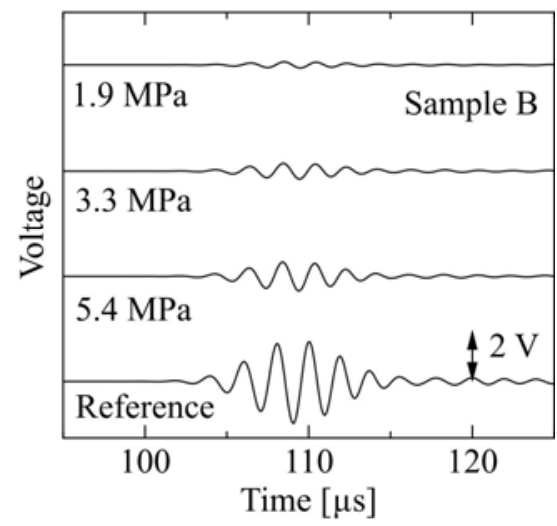

Fig. 2 Measured transmission waveforms of the S0 mode for (a) Sample A and (b) Sample B at different nominal contact pressures, together with that for the reference specimen.

(a)

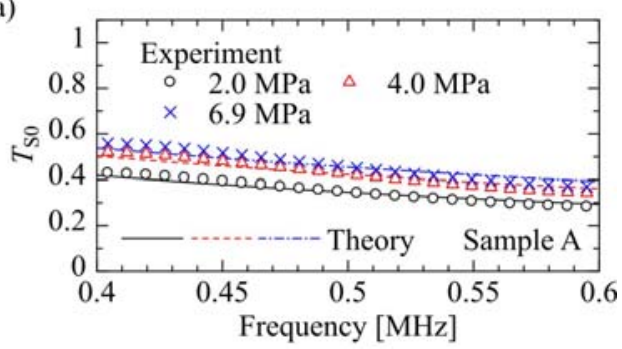

(b)

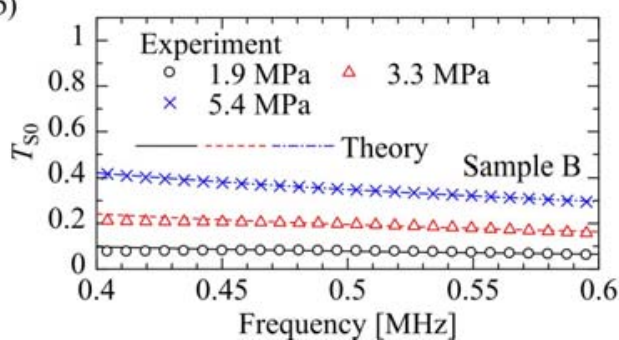

Fig. 3 Frequency dependence of the S0 mode transmission coefficient for (a) Sample A and (b) Sample B at different nominal contact pressures. Curves are drawn from theoretical analysis based on the estimated interfacial stiffnesses. (a)

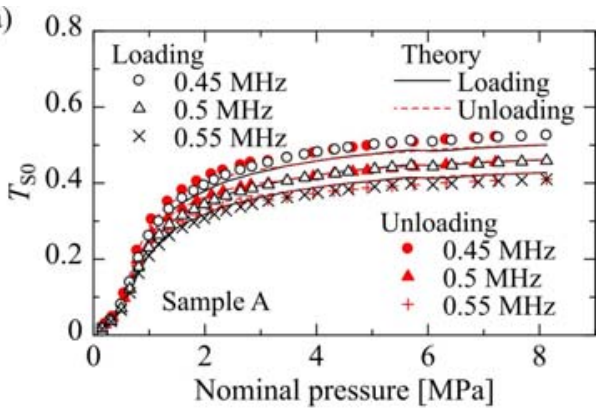

(b)

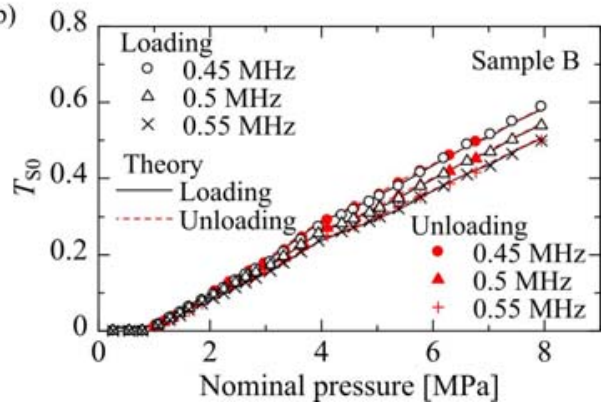

Fig. 4 Contact-pressure dependence of the S0 mode transmission coefficient for (a) Sample A and

(b) Sample B at different frequencies. Curves are drawn from theoretical analysis based on the estimated interfacial stiffnesses. 
(a)

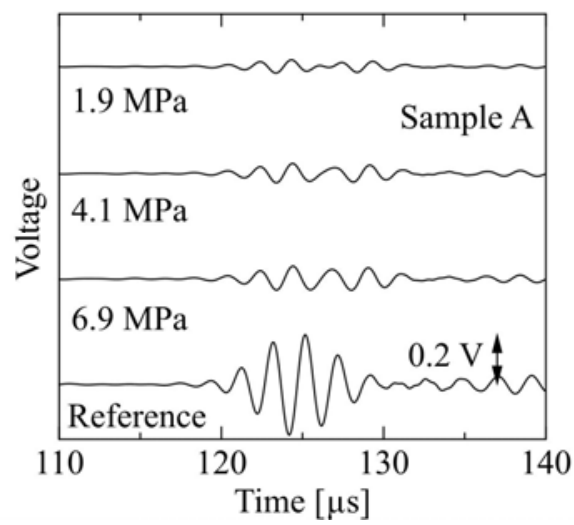

(b)

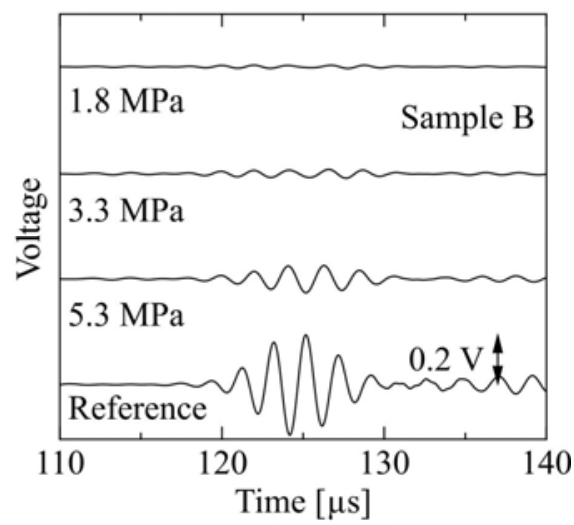

Fig. 5 Measured transmission waveforms of the A0 mode for (a) Sample A and (b) Sample B at different nominal contact pressures, together with that for the reference specimen.

(a)

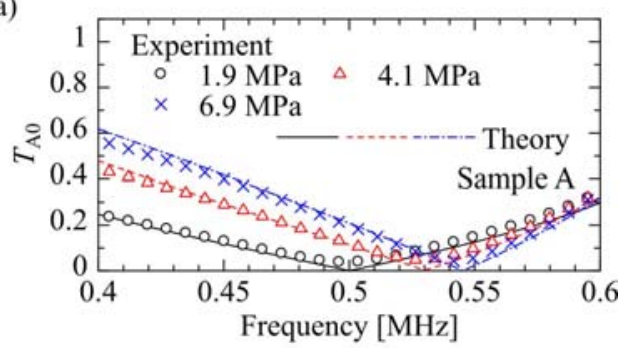

(b)

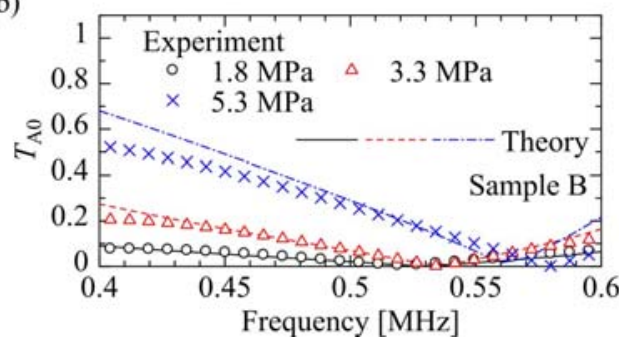

Fig. 6 Frequency dependence of the A0 mode transmission coefficient for (a) Sample A and (b) Sample B at different nominal contact pressures. Curves are drawn from theoretical analysis based on the estimated interfacial stiffnesses. (a)

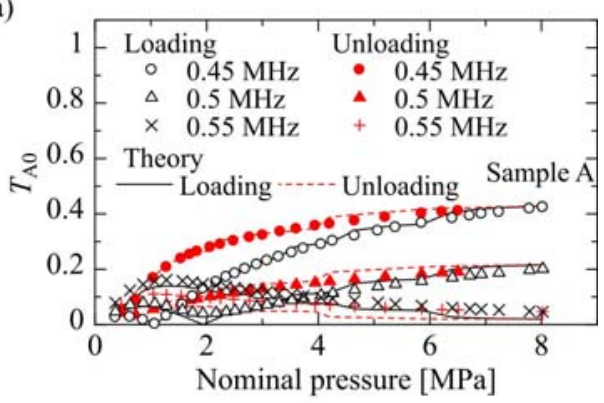

(b)

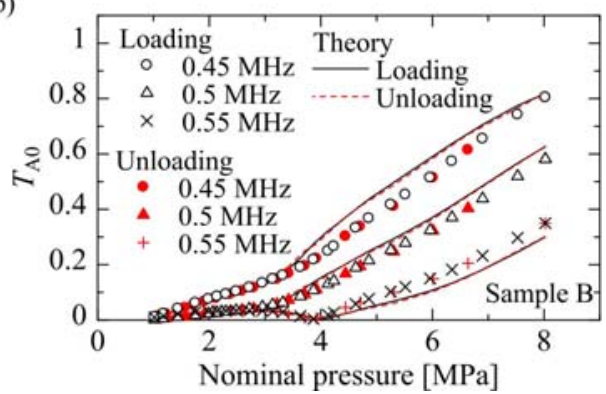

Fig. 7 Contact-pressure dependence of the A0 mode transmission coefficient for (a) Sample A and (b) Sample B at different frequencies. Curves are drawn from theoretical analysis based on the estimated interfacial stiffnesses. 
For the excitation of the $0.5 \mathrm{MHz}$ A0 Lamb modes, the measured transmission waveforms are shown in Fig. 5 , where the main wave packet is at around $125 \mu$ s corresponding to the group velocity of the A0 mode (3.2 $\mathrm{km} / \mathrm{s}$ at $0.5 \mathrm{MHz}$ [18]). Figure 6 shows the frequency dependence of the A0 mode transmission coefficient at several nominal pressures. Unlike the S0 mode, the A0 mode transmission coefficient exhibits non-monotonic behavior with the frequency. In particular, the transmission coefficient appears to take a local minimum close to zero at a certain frequency depending on the nominal pressure $(0.50 \mathrm{MHz}$ for $1.9 \mathrm{MPa}, 0.53 \mathrm{MHz}$ for $4.1 \mathrm{MPa}$, and $0.54 \mathrm{MHz}$ for $6.9 \mathrm{MPa}$, respectively, for Sample A). Such minima of the A0 mode transmission coefficient were also observed for adhesive butt joints of plates [18], and will be discussed from a theoretical point of view in Section 4. The dependence of the A0 mode transmission coefficient on the nominal contact pressure is shown in Fig. 7 at three different frequencies. The contact-pressure dependence is not monotonic in contrast to the result of the S0 mode. For Sample A, the transmission coefficients at $0.45 \mathrm{MHz}$ and at $0.5 \mathrm{MHz}$ take a local minimum at around $1 \mathrm{MPa}$ and at $2 \mathrm{MPa}$, respectively.

\subsection{Resonance at the contacting interface}

For the excitation of the S0 Lamb mode with the center frequency of $0.9 \mathrm{MHz}$, the measured transmission waveforms for two samples are shown in Fig. 8 together with that for the reference specimen. The spectral analysis of the wave packet in Fig. 8 (c) for the reference specimen showed that its spectral peak is located at $0.97 \mathrm{MHz}$, which is higher than the excitation frequency $(0.9 \mathrm{MHz})$ because of the frequency-dependent sensitivity of the receiver with the nominal frequency of $1 \mathrm{MHz}$. In Fig. 8 (c), the main wave packet at around $150 \mu$ s is the S0 mode which has its group velocity $1.8 \mathrm{~km} / \mathrm{s}$ at $0.97 \mathrm{MHz}$ [18]. It is observed that the waveforms transmitted through the contacting interfaces in Fig. 8 (a) and (b) do not retain their original waveform, and exhibit long oscillation tails. This is due to the occurrence of resonance at the contacting edges, as also shown experimentally for adhesive butt joints [18].

In order to examine the frequency content of the long-time oscillations, the waveforms from $160 \mu$ s to $175 \mu$ s were analyzed by FFT with a Hanning window function. The calculated spectra are shown in Fig. 9 with the spectral peaks marked. The vertical axis is normalized by the maximum spectral amplitude of the reference waveform for the same time interval. For each sample, the spectral peaks are located at different frequencies for different nominal pressures. Following Refs. [18, 22], these peak frequencies are regarded as the resonance frequencies of the contacting interfaces. Figure 10 shows the dependence of the obtained resonance frequency on the nominal contact pressure in the loading and unloading processes. The resonance frequency increases with the contact pressure for both samples, but with different pressure-dependence curves. 
(a)

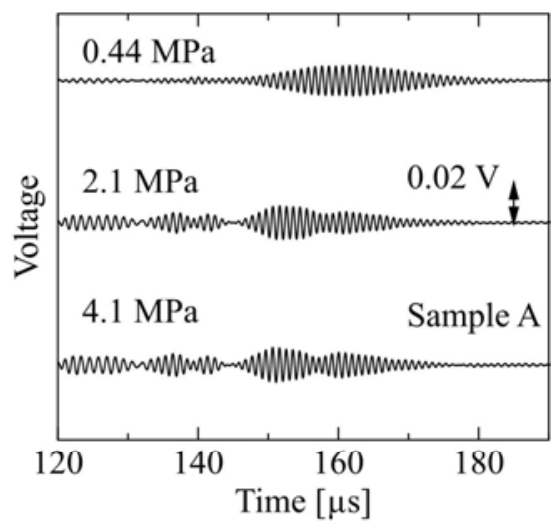

(b)

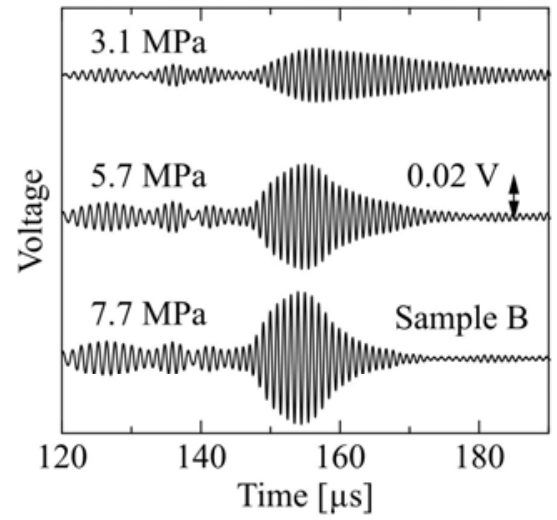

(c)

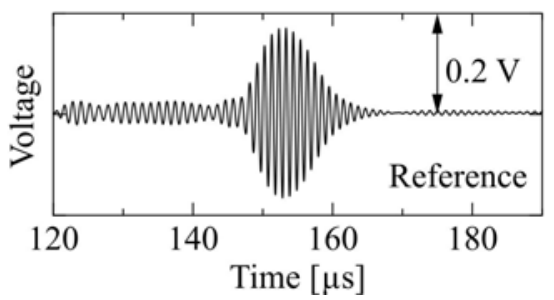

Fig. 8 Measured transmission waveforms of the S0 mode for (a) Sample A and (b) Sample B at different nominal contact pressures, and for (c) the reference specimen. 
(a)

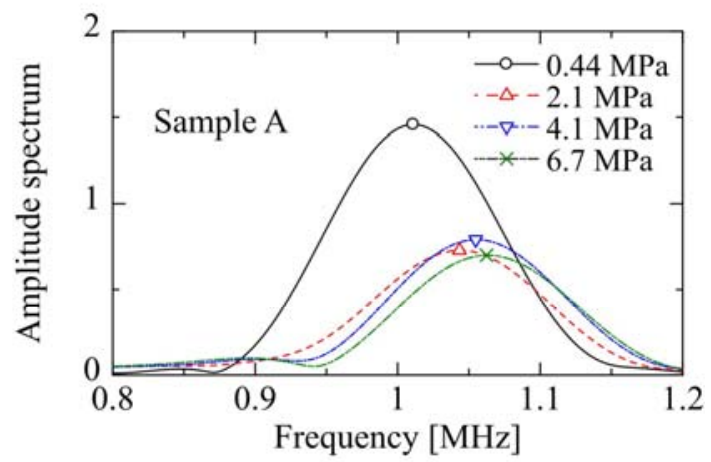

(b)

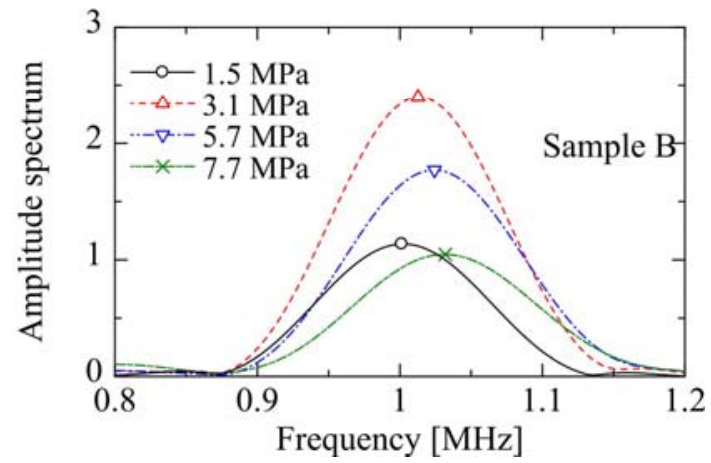

Fig. 9 Amplitude spectra of the transmitted and the reference waveforms from $160 \mu$ s to $175 \mu$ s. The vertical axis is normalized with the maximum amplitude of the reference waveform.

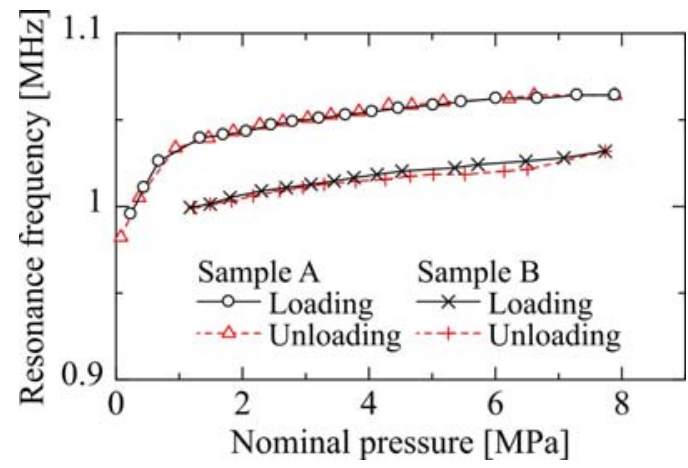

Fig. 10 Contact-pressure dependence of the resonance frequency for Samples A and B. 


\section{Discussions}

\subsection{Estimation of interfacial stiffnesses by the transmission coefficients}

When the contacting edges of plates are modeled as a linear spring-type interface, the amplitude transmission coefficients of the S0 and A0 modes are functions of the frequency and the interfacial stiffnesses [16]. Based on this, the normal and tangential stiffnesses of the contacting interface are estimated from the experimental results shown in Section 3.1. The optimal values of $K_{\mathrm{N}}$ and $K_{\mathrm{T}}$ are obtained by minimizing the squared difference between the measured and the theoretical transmission coefficients summed for the frequency range of $0.45 \mathrm{MHz}$ to $0.55 \mathrm{MHz}$. The thin-plate approximations [16] are used to compute the theoretical transmission coefficients as they are effective for the optimization purpose. It is noted that $K_{\mathrm{N}}$ can be determined from the $\mathrm{S} 0$ mode transmission coefficients alone, since the dependence of the latter on $K_{\mathrm{T}}$ has been found to be negligible [16]. More details of this identification procedure are described in Ref. [18] where it is applied to estimate the interfacial stiffnesses of adhesive butt joints of plates manufactured with different bonding conditions.

The estimated normal and tangential stiffnesses are shown in Fig. 11 for two samples as functions of the nominal contact pressure. The interfacial stiffnesses of Sample A show rapid initial increase with the contact pressure and appear to saturate as the pressure becomes higher. On the other hand, the stiffnesses of Sample B exhibit slower initial increase but continue to increase steadily with the pressure. These features are similar to the contact-pressure dependence of the S0-mode transmission coefficients shown in Fig. 4. It is of some interest to note that the tangential stiffnesses also show monotonic contact-pressure dependence, in spite of the fact that these stiffnesses have been determined from the A0-mode transmission coefficients which were found to depend on the contact pressure in non-monotonic manners as shown in Fig. 7.
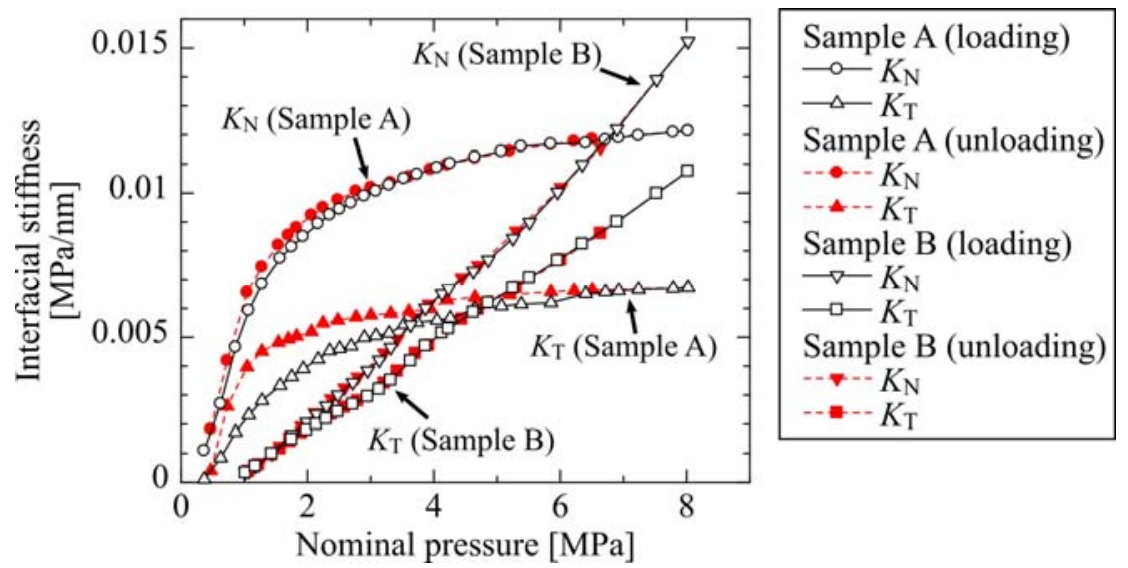

Fig. 11 Contact-pressure dependence of the normal and tangential stiffnesses estimated by the transmission coefficients for Samples A and B.

The theoretical transmission coefficients of the $\mathrm{S} 0$ and $\mathrm{A} 0$ modes calculated by the estimated interfacial stiffnesses and the thin-plate theories were shown in Figs. 3, 4, 6 and 7 for comparison with those obtained experimentally. It can be confirmed that the spring-type interface model well reproduces the experimental results, particularly the presence of minima in the A0 mode transmission coefficients in Figs. 6 and 7. This 
behavior was predicted theoretically in Ref. [16] based on the spring-type interface model combined with the Lamb-wave theory, and also reproduced accurately by the thin-plate theory. This is due to the mutual interaction of the propagating mode (A0 mode) and other non-propagating modes (e.g. A1 mode): in a certain special situation, the motion due to the incident wave is almost completely supported by the non-propagating A1 mode in the plate on the transmission side, which results in negligible transmission of the A0 mode accompanying a minimum of the transmission spectrum.

\subsection{Estimation of interfacial stiffnesses by the resonance frequency}

According to the numerical analysis [17] based on the two-dimensional elastodynamic theory, the imperfect joint of plates modeled as a linear spring-type interface shows resonance behavior at two frequencies in the range $f_{\mathrm{FR}}<f<f_{\mathrm{ZGV}}$ when subjected to the $\mathrm{S} 0$ mode incidence, where $f_{\mathrm{FR}}$ is the resonance frequency of the free edge [23-25] and $f_{\mathrm{ZGV}}$ is the zero-group-velocity frequency of the S1 Lamb mode. For aluminum plates of thickness $2.5 \mathrm{~mm}$, these frequencies are $f_{\mathrm{FR}}=0.95 \mathrm{MHz}$ and $f_{\mathrm{ZGV}}=1.16 \mathrm{MHz}$. It is shown in the numerical analysis [17] that the resonance frequencies increase monotonically with the interfacial stiffnesses.

As shown in Fig. 10, the resonance frequencies obtained in the present measurement are located in the range between $0.95 \mathrm{MHz}$ and $1.16 \mathrm{MHz}$ in agreement with the theory. The present experiment was, however, able to identify only a single resonance frequency at each nominal pressure in contrast to the presence of two frequencies predicted by the theory. The similar situation was observed for adhesive butt joints of plates [18]. This is partly due to the fact that only the parts of waveforms with limited time duration could be used to calculate the spectrum of long-time oscillation, which can make the frequency resolution insufficient for the separate identification of two resonance frequencies.

The analysis in Ref. [17] has also shown that the two resonance frequencies depend on the normal and tangential stiffnesses separately. Furthermore, in certain situations the two resonance frequencies can be very close to each other, and appear to be degenerated as a single resonance frequency. Accounting for these facts, it is tentatively assumed here that the single resonance frequency observed experimentally is a degenerated one, and the normal and tangential stiffnesses are estimated at each pressure from the theoretical relation obtained in Ref. [17]. The estimated stiffnesses are shown in Fig. 12, which show monotonic contact-pressure dependence. The stiffnesses shown in Fig. 12 are, however, remarkably higher than those estimated from the transmission coefficients in Fig. 11.

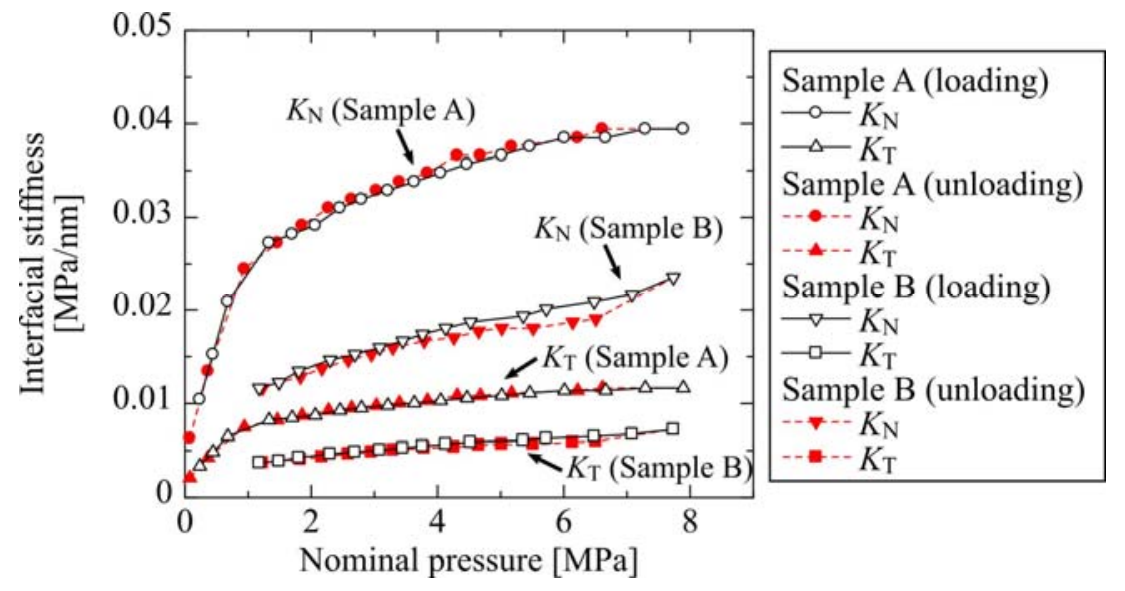

Fig. 12 Contact-pressure dependence of the normal and tangential stiffnesses estimated by the resonance frequency for Samples A and B. 
The difference between the identified interfacial stiffnesses shown in Figs. 11 and 12 may be partly due to some measurement errors or the particular theoretical assumptions, but is worthy of due attention from the following point of view. The two results of interfacial stiffnesses have been obtained in different ranges of frequency, i.e., from the transmission coefficients for the frequency range centered at $0.5 \mathrm{MHz}$, and from the resonance frequencies at around $1 \mathrm{MHz}$. Therefore, the results indicate the possible frequency dependence of interfacial stiffnesses. In a previous work [14], the interfacial stiffnesses of the contacting interfaces of aluminum alloy blocks were measured using ultrasonic bulk and interface waves. Their results also suggested the presence of significant frequency dependence of the interfacial stiffnesses: the tangential stiffness measured from the interface wave velocity showed values below $0.01 \mathrm{MPa} / \mathrm{nm}$ for the pressure up to $4 \mathrm{MPa}$ at the frequency of $0.5 \mathrm{MHz}$, while the tangential stiffness measured from the shear wave reflection coefficient was about $0.25 \mathrm{MPa} / \mathrm{nm}$ at $4 \mathrm{MPa}$ and at the frequency of $2.25 \mathrm{MHz}$. In Ref. [14], two possible sources of such frequency dependence were examined, namely, the spatial inhomogeneity of contact conditions as well as the dissipative behavior of the interface. The frequency dependence of interfacial stiffnesses of contacting interfaces has also been discussed in the context of geophysical study of fractures [26, 27].

\subsection{Remark on the characterization of contact condition of plates using Lamb waves}

The present study has shown that the transmission coefficients of the S0 and A0 Lamb modes reflect the contact condition of the plate edges. The S0 mode transmission coefficient depends monotonically on the contact pressure and the frequency in the similar fashion to bulk waves [9-14]. This nature makes the S0 mode transmission coefficient a convenient indicator of the contact condition. On the other hand, the A0 mode transmission coefficient may be more difficult to use as a simple indicator, as its dependence on the contact pressure and the frequency is not monotonic. In Ref. [18], it was shown that the quality of adhesive butt joints of plates can be characterized by the interfacial stiffnesses obtained from the $\mathrm{S} 0$ and $\mathrm{A} 0$ mode transmission coefficients. In an on-going investigation, the Lamb wave propagation characteristics on a plate with a fatigue crack is being examined by applying the spring-type interface model to the crack faces, which will be reported elsewhere.

The resonance frequency has also been found to show similar contact-pressure dependence as the transmission coefficients. Namely, the resonance frequency of Sample A shows rapid initial increase in the low pressure range, while that of Sample B shows gradual increase with the applied pressure. Consequently, the resonance frequencies also reflect the nature of the contacting edges. The resonance signal in the transmission waveform can be, however, very weak as can be seen in Fig. 8. In order to examine the intensity as well as the duration of the resonance signal, the magnitude of the spectral peaks in Fig. 9 is plotted against the nominal contact pressure in Fig. 13. For Sample A, the peak amplitude is highest for the weakest contact and decreases to low levels for higher contact pressures. For Sample B, it is first unquantifiable at low pressures, then increases with the contact pressure to attain its maximum at about $3 \mathrm{MPa}$, and finally starts to decrease with further increase of the contact pressure. Such trends can be understood as follows: (I) at very low contact pressures, the transmitted wave is weak, so the resonance signal is also weak, (II) as the contact pressure increases, the transmitted wave becomes stronger, accompanied by higher resonance signal, and (III) as the contact pressure increases further, the joint approaches perfect contact (no interface), so the joint resonance tends to diminish. Clearly, Sample B follows this trend. For Sample A, the presence of the stages (I) and (II) is unclear since the interface attains sufficiently high stiffnesses with very low contact pressures, leaving only the stage (III) observed experimentally. When Fig. 13 is combined with the results in Fig. 12, it is seen that the peak amplitude in Fig. 13 is higher than unity when the normal stiffness of the contacting interface is roughly below $0.02 \mathrm{MPa} / \mathrm{nm}$ for both samples. From this observation, the resonant oscillation can be detected more easily and can serve as an indicator of contact condition when the contact of plate edges is relatively weak. 


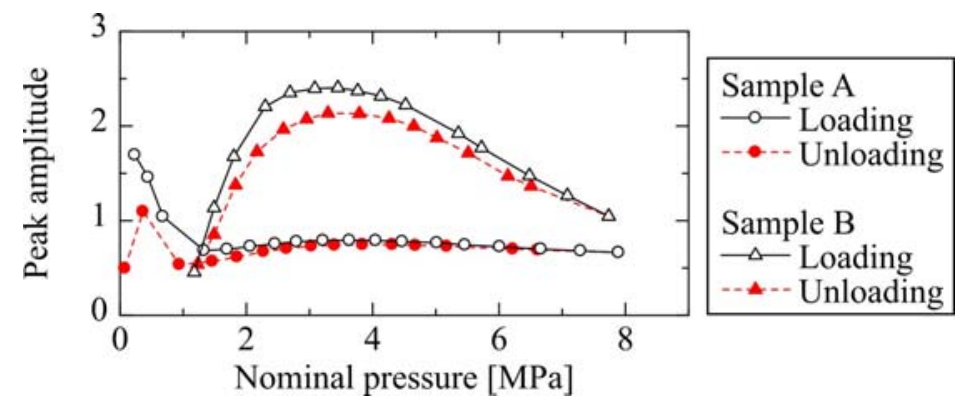

Fig. 13 Contact-pressure dependence of the spectral peak amplitude of the long-time oscillation in the transmission waveform for Samples A and B.

\section{Conclusion}

In this study, the interaction of the S0 and A0 Lamb waves with contacting edges of two aluminum alloy plates has been investigated experimentally. The transmission coefficient of the S0 mode increases monotonically with nominal contact pressure, while the transmission coefficient of the A0 mode takes a minimum at specific frequency and contact pressure. The resonance frequency of the contacting edges has been found to increase monotonically with the contact pressure. The normal and tangential stiffnesses of the contacting interface obtained based on the spring-type interface model increase with the contact pressure. The difference between the interfacial stiffnesses identified from the transmission coefficients and those from the resonance frequency indicates their frequency dependence. The present study shows that the S0 mode transmission coefficient can be a particularly convenient indicator of the contact condition of plate edges. The resonance frequency may also serve as a parameter to indicate a weak contact of plate edges.

\section{Acknowledgements}

This work has been supported by JSPS KAKENHI Grant Numbers 26-2190 and 25289005.

\section{References}

[1] S. Rohklin, Diffraction of Lamb waves by a finite crack in an elastic layer, J. Acoust. Soc. Am. 67 (1980), $1157-1165$.

[2] D. Alleyne, P. Cawley, The interaction of Lamb waves with defects, IEEE Trans. Ultrason. Ferroelectr. Freq. Control 39 (1992), 381-397.

[3] Y. Cho, D. D. Hongerholt, J. L. Rose, Lamb wave scattering analysis for reflector characterization, IEEE Trans. Ultrason. Ferroelectr. Freq. Control 44 (1997), 44-52.

[4] M. J. S. Lowe, O. Diligent, Low-frequency reflection characteristics of the $s_{0}$ Lamb wave from a rectangular notch in a plate, J. Acoust. Soc. Am. 111 (2002), 64-74.

[5] R. Benz, M. Niethammer, S. Hurlebaus, L. J. Jacobs, Localization of notches with Lamb waves, J. Acoust. Soc. Am. 114(2003), 677-685.

[6] J.-B. Ihn, F.-K. Chang, Detection and monitoring of hidden fatigue crack growth using a built-in piezoelectric sensor/actuator network: I. Diagnostics, Smart Mater. Struct. 13 (2004), 609-620.

[7] M. Ryles, F. H. Ngau, I. McDonald, W. J. Staszewski, Comparative study of nonlinear acoustic and Lamb wave techniques for fatigue crack detection in metallic structures, Fatigue Fract. Eng. Mater. Struct. 31 (2008), 674-683. 
[8] H. Cho and C. J. Lissenden, Structural health monitoring of fatigue crack growth in plate structures with ultrasonic guided waves, Struct. Health Monit. 11 (2011), 393-404.

[9] J.-M. Baik, R. B. Thompson, Ultrasonic scattering from imperfect interfaces: A quasi-static model, J. Nondestruct. Eval. 4 (1984), 177-196.

[10] A. Boström, G. Wickham, On the boundary conditions for ultrasonic transmission by partially closed cracks, J. Nondestruct. Eval. 10 (1991), 139-149.

[11] P. B. Nagy, Ultrasonic classification of imperfect interfaces, J. Nondestruct. Eval. 11 (1992) 127-139.

[12] B. W. Drinkwater, R. S. Dwyer-Joyce, P. Cawley, A study of the interaction between ultrasound and a partially contacting solid-solid interface, Proc. R. Soc. London, Ser. A 452 (1996) 2613-2628.

[13] D. Liaptsis, B. W. Drinkwater, R. Thomas, The interaction of oblique incidence ultrasound with rough, partially contacting interfaces, Nondestruct. Tes. Eval. 21 (2006) 109-121.

[14] S. Biwa, S. Hiraiwa, E. Matsumoto, Stiffness evaluation of contacting surfaces by bulk and interface waves, Ultrasonics 47 (2007) 123-129.

[15] C. Pecorari, Scattering of a Rayleigh wave by a surface-breaking crack with faces in partial contact, Wave Motion 33 (2001) 259-270.

[16] N. Mori, S. Biwa, T. Hayashi, Reflection and transmission of Lamb waves at an imperfect joint of plates, J. Appl. Phys. 113 (2013) 074901.

[17] N. Mori, S. Biwa, Resonance of an imperfect joint of plates by the lowest-order symmetric Lamb mode, J. Acoust. Soc. Am. 137 (2015) 3139-3148.

[18] N. Mori, S. Biwa, Transmission of Lamb waves and resonance at an adhesive butt joint of plates, Ultrasonics 72 (2016) 80-88.

[19] T. Kundu, A. Maji, T. Ghosh, K. Maslov, Detection of kissing bonds by Lamb waves, Ultrasonics 35 (1998), 573-580.

[20] B. W. Drinkwater, M. Castaings, B. Hosten, The measurement of $A_{0}$ and $S_{0}$ Lamb wave attenuation to determine the normal and shear stiffnesses of a compressively loaded interface, J. Acoust. Soc. Am. 113 (2003), 3161-3170.

[21] A. Balvantín, A. Baltazar, J. I. Aranda-Sanchez, A study of guided wave propagation on a plate between solid bodies with imperfect boundary conditions, Int. J. Mech. Sci. 63 (2012), 66-73.

[22] N. Mori, S. Biwa, Interaction of Lamb waves with an imperfect joint of plates: reflection, transmission and resonance, Phys. Procedia 70 (2015) 480-483.

[23] P. J. Torvik, Reflection of wave trains in semi-infinite plates, J. Acoust. Soc. Am. 41 (1967) 346-353.

[24] E. Le Clezio, M. V. Predoi, M. Castaings, B. Hosten, M. Rousseau, Numerical predictions and experiments on the free-plate edge mode, Ultrasonics 41 (2003) 25-40.

[25] V. Pagneux, Revisiting the edge resonance for Lamb waves in a semi-infinite plate, J. Acoust. Soc. Am. 120 (2006) 649-656.

[26] L. Prrak-Nolte, D. D. Nolte, Frequency dependence of fracture stiffness, Geophys. Res. Lett. 19 (1992) 325-328.

[27] A. F. Baird, J.-M. Kendall, D. A. Angus, Frequency-dependent seismic anisotropy due to fractures: fluid flow versus scattering, Geophysics 78 (2013) WA111-WA122. 


\section{Figure captions}

Fig. 1 Schematic of the experimental setup.

Fig. 2 Measured transmission waveforms of the S0 mode for (a) Sample A and (b) Sample B at different nominal contact pressures, together with that for the reference specimen.

Fig. 3 Frequency dependence of the S0 mode transmission coefficient for (a) Sample A and (b) Sample B at different nominal contact pressures. Curves are drawn from theoretical analysis based on the estimated interfacial stiffnesses.

Fig. 4 Contact-pressure dependence of the S0 mode transmission coefficient for (a) Sample A and (b) Sample $\mathrm{B}$ at different frequencies. Curves are drawn from theoretical analysis based on the estimated interfacial stiffnesses.

Fig. 5 Measured transmission waveforms of the A0 mode for (a) Sample A and (b) Sample B at different nominal contact pressures, together with that for the reference specimen.

Fig. 6 Frequency dependence of the A0 mode transmission coefficient for (a) Sample A and (b) Sample B at different nominal contact pressures. Curves are drawn from theoretical analysis based on the estimated interfacial stiffnesses.

Fig. 7 Contact-pressure dependence of the A0 mode transmission coefficient for (a) Sample A and (b) Sample B at different frequencies. Curves are drawn from theoretical analysis based on the estimated interfacial stiffnesses.

Fig. 8 Measured transmission waveforms of the S0 mode for (a) Sample A and (b) Sample B at different nominal contact pressures, and for (c) the reference specimen.

Fig. 9 Amplitude spectra of the transmitted and the reference waveforms from $160 \mu$ s to $175 \mu$ s. The vertical axis is normalized with the maximum amplitude of the reference waveform.

Fig. 10 Contact-pressure dependence of the resonance frequency for Samples A and B.

Fig. 11 Contact-pressure dependence of the normal and tangential stiffnesses estimated by the transmission coefficients for Samples A and B.

Fig. 12 Contact-pressure dependence of the normal and tangential stiffnesses estimated by the resonance frequency for Samples A and B.

Fig. 13 Contact-pressure dependence of the spectral peak amplitude of the long-time oscillation in the transmission waveform for Samples A and B. 